\title{
Avaliação da força muscular de flexores e extensores de joelho em indivíduos idosos socialmente ativos
}

\author{
Lia Mara Wibelinger*; Rodolfo Herberto Schneider ${ }^{\star *}$; Aline Tonial ${ }^{* \star *}$; \\ Giseli Oliveira ${ }^{\star * *}$; Bruna Klein ${ }^{\star * *}$; Debora Capitânio***
}

\section{Resumo}

O envelhecimento traz consigo várias manifestações fisiológicas, dentre as quais a perda de força muscular, assim como da habilidade do músculo para exercer força rapidamente. A partir dos sessenta anos de idade esta perda se torna mais severa e é responsável por limitações na funcionalidade, déficits de equilíbrio e risco de quedas. Esta pesquisa teve como objetivo avaliar a força muscular (torque muscular) de flexores e extensores de joelho em indivíduos idosos socialmente ativos. Participaram da pesquisa cem indivíduos idosos socialmente ativos nas faixas etárias entre 60 e 87 anos de idade, de ambos os sexos, que frequentavam o Centro Regional de Estudos Aplicados à Terceira Idade da Universidade de Passo Fundo, no município de Passo Fundo - RS, no período de março a junho de 2007. Foram excluídos do estudo os indivíduos que apresentavam déficit cognitivo e que não conseguiam realizar flexão de joelho. Para a realização desta pesquisa foi utilizado o dinamômetro isocinético Biodex Multi Joint 3, nas velocidades de $120^{\circ}$, $180^{\circ}$ e $240^{\circ}$; nos movimentos de flexão e extensão. Inicialmente, os participantes realizaram um aquecimento de $5 \mathrm{~min}$ em bicicleta ergométrica e após foram submetidos a uma série de três repetições dos movimentos, sendo considerada a média das três repetições. As avaliações aconteceram no Laboratório de Biomecânica da Faculdade de Educação Física e Fisioterapia da Universidade de Passo Fundo. Neste estudo foi possível observar que na comparação entre os membros inferiores direito e esquerdo os desequilíbrios musculares somente ultrapassaram $10 \%$ na amostra com mais de oitenta anos, no sexo masculino, o que não se pode considerar por esta amostra ser composta somente por dois indivíduos em cada gênero. $\mathrm{Na}$ média do pico de torque dos indivíduos do sexo masculino foi possível verificar a significância do movimento de flexão a $240^{\circ}$ com $p<0,03$, quando analisada toda a amostra, e nos indivíduos de 60-69 anos, no movimento de extensão a $120^{\circ}$ com $p<0,04$. Ao comparar os diferentes gêneros, verifica-se que nos indivíduos do sexo masculino estão os picos de torque mais elevados. A força muscular (torque muscular) é maior nos indivíduos do sexo masculino; os músculos extensores do joelho (quadríceps) são os mais fortes. Os músculos flexores não apresentam relação entre velocidade e pico de torque. A presença de doença osteoarticular, principalmente nas mulheres, pode ter influenciado no menor pico de torque destes indivíduos.

Palavras-chaves: Envelhecimento. Força muscular. Torque. Dinamômetro de força muscular.

* Mestra e doutoranda em Gerontologia Biomédica pela Pontifícia Universidade Católica do Rio Grande do Sul. Professora da Faculdade de Educação Física e Fisioterapia da Universidade de Passo Fundo. Endereço para correspondência: Rua Uruguai, 2200, CEP 99010-112, Passo Fundo - RS. E-mail: liafisio@upf.br.

** Médico. Doutor em Medicina e Ciências da Saúde pela Pontifícia Universidade Católica do Rio Grande do Sul. Professor do Programa de Pós-Graduação em Gerontologia Biomédica da pela Pontifícia Universidade Católica do Rio Grande do Sul.

**** Acadêmicas do curso de Fisioterapia da Universidade de Passo Fundo.

$\longrightarrow$ Recebido em fevereiro de 2009 - Avaliado em março de 2010.

$\hookrightarrow$ doi:10.5335/rbceh.2009.027 


\section{Introdução}

Dentre os vários órgãos afetados pelo processo de envelhecimento, a musculatura esquelética é a que, provavelmente, influencia de forma mais significativa as alterações na qualidade de vida dos indivíduos. É importante o conhecimento das alterações funcionais e estruturais dos músculos esqueléticos que ocorrem nesse processo, a fim de distinguir entre as alterações patológicas e fisiológicas. Nas últimas décadas, a necessidade deste conhecimento tornou-se maior em razão do aumento da expectativa de vida e do consequente aumento da população idosa. (CAROMANO; TSAI, 1999).

Relata-se um declínio em torno de $15 \%$ entre a sexta e a sétima décadas e, após a sétima década, um declínio de cerca de $30 \%$ da força máxima individual a cada década. (REBELATO; MORELLI, 2004).

As comparações bilaterais são feitas para quantificar possíveis déficits entre a musculatura do mesmo grupo muscular, de forma bilateral, isto é, no caso de joelho, avaliar comparativamente o grupo responsável pela flexão de joelho e o grupo responsável pela extensão de joelho. (DVIR, 2002).

A temática e os objetivos centrais desta pesquisa estão baseados no fato de que a perda de força muscular de membro inferior é fator de limitação para a realização das atividades de vida diária, mobilidade e quedas nos indivíduos idosos. Por essa razão, optou-se pelo uso de uma avaliação isocinética, visto que a utilização deste método é sugerida em pesquisas com indivíduos idosos.
O presente estudo avaliou a força muscular (torque muscular) de flexores e extensores de joelhos por meio do uso do dinamômetro isocinético computadorizado Biodex TM Multi Joint System 3 Pro, nas velocidades angulares de 120 , 180 e 240 graus/s, em indivíduos idosos socialmente ativos.

\section{Metodologia}

O estudo caracteriza-se como quantitativo, comparativo, de cunho transversal sobre a força muscular de flexores e extensores de joelho em indivíduos idosos socialmente ativos. Incluiu mil indivíduos idosos socialmente ativos na faixa etária entre 60 e 95 anos de idade, participantes do Centro Regional de Estudos Sobre a Terceira Idade da Universidade de Passo Fundo (Creati). A amostra foi composta por cem indivíduos idosos socialmente ativos na faixa etária entre 60 e 87 anos de idade, participantes do Creati.

Para a realização deste estudo foi utilizado o dinamômetro isocinético computadorizado Biodex TM Multi Joint System 3 Pro, nas velocidades angulares de 120,180 e 240 graus/s. Por meio da avaliação física foi estabelecido o primeiro contato com os voluntários e foram coletados os dados pessoais e informações sobre suas queixas, doenças associadas, prática de atividade física e se estavam fazendo uso de medicamentos. Após foi realizado um pré-aquecimento de 5 ' em bicicleta eletromagnética Movement BM 2700, sem carga, com assento em altura adequada. Para segurança na realização do teste foram aferidas a pressão arterial e a frequência cardíaca anteriormente ao 
teste e imediatamente após cada série, sendo a série seguinte realizada após três minutos de descanso, para que esses parâmetros voltassem aos valores de repouso e a fadiga não comprometesse a eficiência do teste.

A fim de atender aos objetivos propostos para o estudo, as informações coletadas foram tratadas estatisticamente mediante o uso do pacote computadorizado Statistica for Windows, adotando-se o seguinte procedimento: para comparar a força muscular entre os gêneros foi utilizado o teste "t" de Student para amostras independentes e a análise de regressão a fim de relacionar a idade com o torque dos indivíduos avaliados.

O estudo foi realizado após a apreciação e aprovação da Comissão Científica do Instituto de Geriatria e Gerontologia da Pontifícia Universidade Católica do Rio Grande do Sul e do Comitê de Ética em Pesquisa da PUCRS e seguiu as recomendações da resolução 196/1996 do Conselho Nacional de Saúde, sendo aprovado em 18 de setembro de $2006 \mathrm{sob}$ registro CEP 06/03349. Os indivíduos participantes do estudo assinaram o termo de consentimento livre e esclarecido.

\section{Resultados}

Um total de cem indivíduos idosos foi incluído no estudo, sendo $81 \%$ do sexo feminino. A maioria dos participantes (84\%) faz uso de medicação, principalmente para hipertensão arterial sistêmica $(66 \%)$, e realiza atividade física regularmente $(78 \%)$, especialmente a hidroginástica (59\%).

A Tabela 1 apresenta a média dos valores do pico de torque (momento) dos indivíduos do sexo masculino. É possível observar que no movimento de flexão a uma velocidade de $240^{\circ}$ existe uma significância de $p=0,03$. No teste de regressão $\left(\mathrm{R}^{2}\right)$ os valores são considerados relevantes, pois as variáveis em análise, além da idade mais avançada, possuem o pico de torque também alto; por isso, há uma relação entre as variáveis, mostrando a característica masculina com alto pico de torque.

Tabela 1 - Pico de torque (momento) do grupo de homens avaliados.

\begin{tabular}{|c|c|c|c|c|c|}
\hline Velocidade & Movimento & JD (Nm) & JE(Nm) & p-valor & Valor $\mathrm{R}^{2}$ \\
\hline \multirow{2}{*}{$120^{\circ}$} & Flexão & $38,5 \pm 17,7$ & $39,1 \pm 19,5$ & 0,37 & 44,0 \\
\hline & Extensão & $83,3 \pm 42,7$ & $87,0 \pm 40,0$ & 0,21 & 51,2 \\
\hline \multirow{2}{*}{$180^{\circ}$} & Flexão & $38,1 \pm 13,8$ & $38,8 \pm 17,1$ & 0,33 & 66,8 \\
\hline & Extensão & $74,5 \pm 33,1$ & $77,4 \pm 31,5$ & 0,19 & 49,2 \\
\hline \multirow{2}{*}{$240^{\circ}$} & Flexão & $35,1 \pm 14,6$ & $38,8 \pm 15,7$ & 0,03 & 28,1 \\
\hline & Extensão & $65.2 \pm 24.0$ & $63,5 \pm 26,2$ & 0,31 & 44,6 \\
\hline
\end{tabular}

A Tabela 2 apresenta a amostra dos indivíduos do sexo feminino, mostrando diferenças entre as velocidades e movimentos, porém não estatisticamente significativas. Ao comparar as tabelas 2 e 3 , é possível verificar que houve diferença significativa somente no movimento de flexão a $240^{\circ}$ com $\mathrm{p}=0,03$ na Tabela 2 , que apresenta o pico de torque dos indivíduos do sexo masculino. Nos indi- 
víduos do sexo feminino os valores do $\mathrm{R}^{2}$ amostra masculina, sendo as médias dos são menores quando comparados com a picos de torque também menores.

Tabela 2 - Pico de torque (momento) do grupo de mulheres avaliados.

\begin{tabular}{|c|c|c|c|c|c|}
\hline Velocidade & Movimento & JD (Nm) & $\mathrm{JE}(\mathrm{Nm})$ & $p$-valor & Valor $\mathrm{R}^{2}$ \\
\hline \multirow{2}{*}{$120^{\circ}$} & Flexão & $26,2 \pm 11,9$ & $26,7 \pm 11,8$ & 0,28 & 3,7 \\
\hline & Extensão & $55,0 \pm 22,0$ & $54,4 \pm 21,4$ & 0,35 & 4,6 \\
\hline \multirow{2}{*}{$180^{\circ}$} & Flexão & $24,6 \pm 9,9$ & $25,4 \pm 10,8$ & 0,18 & 9,8 \\
\hline & Extensão & $46,5 \pm 17,9$ & $46,3 \pm 17,2$ & 0,43 & 2,2 \\
\hline \multirow{2}{*}{$240^{\circ}$} & Flexão & $25,8 \pm 10,3$ & $25,7 \pm 10,3$ & 0,46 & 7,8 \\
\hline & Extensão & $41,8 \pm 14,2$ & $41,8 \pm 14,1$ & 0,33 & 4,6 \\
\hline
\end{tabular}

Na Tabela 3 é possível observar que significativas em nenhuma velocidade não houve diferenças estatisticamente e em nenhuma faixa etária.

Tabela 3 - Pico de torque (momento) do grupo de mulheres avaliados por faixa etária.

\begin{tabular}{|c|c|c|c|c|c|c|}
\hline Faixa etária & Velocidade & Movimento & JD (Nm) & $\mathrm{JE}(\mathrm{Nm})$ & $\mathrm{p}$-valor & Valor $\mathrm{R}^{2}$ \\
\hline \multirow{6}{*}{$60-69$ anos } & \multirow{2}{*}{$120^{\circ}$} & Flexão & $27,0 \pm 12,7$ & $28,0 \pm 11,4$ & 0,35 & 1,2 \\
\hline & & Extensão & $57,0 \pm 23,3$ & $58,6 \pm 20,4$ & 0,48 & 2,3 \\
\hline & \multirow{2}{*}{$180^{\circ}$} & Flexão & $25,2 \pm 10,2$ & $26,9 \pm 10,8$ & 0,09 & 4,2 \\
\hline & & Extensão & $47,3 \pm 19,3$ & $49,6 \pm 17,2$ & 0,31 & 0,2 \\
\hline & \multirow{2}{*}{$240^{\circ}$} & Flexão & $27,1 \pm 11,0$ & $27,3 \pm 10,7$ & 0,88 & 1,1 \\
\hline & & Extensão & $42,8 \pm 15,6$ & $43,6 \pm 14,6$ & 0,59 & 1,0 \\
\hline \multirow{5}{*}{ 70-79 anos } & \multirow[t]{2}{*}{$120^{\circ}$} & Flexão & $22,7 \pm 10,0$ & $22,6 \pm 13,2$ & 0,65 & 2,5 \\
\hline & & Extensão & $51,7 \pm 19,4$ & $47,0 \pm 22,1$ & 0,06 & 9,5 \\
\hline & \multirow[t]{2}{*}{$180^{\circ}$} & Flexão & $22,6 \pm 8,9$ & $22,0 \pm 10,7$ & 0,62 & 1,1 \\
\hline & & Extensão & $43,7 \pm 15,2$ & $41,0 \pm 15,2$ & 0,10 & 0,9 \\
\hline & \multirow[t]{2}{*}{$240^{\circ}$} & Flexão & $23,0 \pm 8,5$ & $22,4 \pm 9,3$ & 0,67 & 0,5 \\
\hline \multirow{7}{*}{$\begin{array}{l}\text { Mais de } 80 \\
\text { anos }^{*}\end{array}$} & & Extensão & $37,6 \pm 12,1$ & $36,9 \pm 13,1$ & 0,63 & 4,3 \\
\hline & \multirow[t]{2}{*}{$120^{\circ}$} & Flexão & $27,1 \pm 5,4$ & $23,7 \pm 7,3$ & 0,51 & \\
\hline & & Extensão & $56,2 \pm 5,3$ & $52,3 \pm 13,6$ & 0,52 & \\
\hline & \multirow[t]{2}{*}{$180^{\circ}$} & Flexão & $20,3 \pm 12,4$ & $17,9 \pm 9,1$ & 0,73 & \\
\hline & & Extensão & $45,2 \pm 6,9$ & $43,2 \pm 12,1$ & 0,63 & \\
\hline & \multirow[t]{2}{*}{$240^{\circ}$} & Flexão & $20,8 \pm 11,8$ & $19,7 \pm 14,5$ & 0,53 & \\
\hline & & Extensão & $38,2 \pm 4,9$ & $41,1 \pm 10,5$ & 0,49 & \\
\hline
\end{tabular}

Nota: *A análise de regressão não foi utilizada na amostra de mais de oitenta anos, pois o n foi baixo. 
A Tabela 4 demonstra os valores do pico de torque das diferentes faixas etárias dos indivíduos do sexo masculino, observando-se que na velocidade de $120^{\circ}$ no movimento de extensão existe dife- rença estatisticamente significativa em ambos os joelhos $(p=0,04)$. Da mesma forma, é observada diferença estatisticamente significativa na velocidade de $180^{\circ}$ no movimento de flexão $(\mathrm{p}=0,03)$.

Tabela 4 - Pico de torque (momento) do grupo de homens avaliados por faixa etária.

\begin{tabular}{|c|c|c|c|c|c|c|}
\hline Faixa etária & Velocidade & Movimento & JD (Nm) & $\mathrm{JE}(\mathrm{Nm})$ & $\mathrm{p}$-valor & Valor $\mathrm{R}^{2}$ \\
\hline \multirow{6}{*}{ 60-69 anos } & \multirow{2}{*}{$120^{\circ}$} & Flexão & $43,4 \pm 15,6$ & $43,4 \pm 18,2$ & 1,00 & 48,5 \\
\hline & & Extensão & $91,1 \pm 44,5$ & $100,1 \pm 41,1$ & 0,04 & 25,3 \\
\hline & \multirow{2}{*}{$180^{\circ}$} & Flexão & $42,1 \pm 11,1$ & $41,1 \pm 16,2$ & 0,62 & 34,0 \\
\hline & & Extensão & $81,4 \pm 34,1$ & $87,7 \pm 30,8$ & 0,12 & 19,3 \\
\hline & \multirow{2}{*}{$240^{\circ}$} & Flexão & $37,2 \pm 12,8$ & $42,2 \pm 13,5$ & 0,07 & 42,2 \\
\hline & & Extensão & $69,3 \pm 25,4$ & $73,9 \pm 23,8$ & 0,27 & 30,4 \\
\hline \multirow{6}{*}{ 70-79 anos } & \multirow[t]{2}{*}{$120^{\circ}$} & Flexão & $37,8 \pm 17,8$ & $40,3 \pm 19,1$ & 0,33 & 2,7 \\
\hline & & Extensão & $80,5 \pm 40,6$ & $76,6 \pm 20,2$ & 0,78 & 18,4 \\
\hline & \multirow[t]{2}{*}{$180^{\circ}$} & Flexão & $37,9 \pm 14,8$ & $41,0 \pm 19,2$ & 0,19 & 23,2 \\
\hline & & Extensão & $73,8 \pm 28,0$ & $73,6 \pm 14,8$ & 0,98 & 10,4 \\
\hline & \multirow[t]{2}{*}{$240^{\circ}$} & Flexão & $41,1 \pm 15,0$ & $40,2 \pm 18,4$ & 0,77 & 4,6 \\
\hline & & Extensão & $69,4 \pm 14,7$ & $55,0 \pm 18,6$ & 0,09 & 13,5 \\
\hline \multirow{6}{*}{$\begin{array}{c}\text { Mais de } 80 \\
\text { anos }^{*}\end{array}$} & \multirow[t]{2}{*}{$120^{\circ}$} & Flexão & $15,1 \pm 3,8$ & $19,8 \pm 4,6$ & 0,07 & \\
\hline & & Extensão & $33,1 \pm 10,8$ & $30,8 \pm 7,1$ & 0,54 & \\
\hline & \multirow[t]{2}{*}{$180^{\circ}$} & Flexão & $17,7 \pm 5,4$ & $24,4 \pm 5,0$ & 0,03 & \\
\hline & & Extensão & $33,6 \pm 5,2$ & $31,1 \pm 8,5$ & 0,49 & \\
\hline & \multirow[t]{2}{*}{$240^{\circ}$} & Flexão & $17,0 \pm 3,7$ & $26,2 \pm 0,7$ & 0,14 & \\
\hline & & Extensão & $36,3 \pm 6,9$ & $28,4 \pm 1,0$ & 0,39 & \\
\hline
\end{tabular}

Nota: *A análise de regressão não foi utilizada na amostra de mais de oitenta anos, pois o $\mathrm{n}$ foi baixo.

\section{Discussão dos resultados}

Segundo Lynch et al. (1999), a diminuição da força dos membros inferiores com a idade é mais acentuada do que a observada nos membros superiores. As velocidades de 120, 180 e 240 graus/s, as quais são consideradas a média e alta velocidade angular, respectivamente, foram adotadas para a realização deste estudo, pois, segundo Dvir (2002), é contraindicado o uso de velocidades muito 
baixas em distúrbios patelofemorais ou ligamentares. Como não há referência prévia da população estudada, optou-se por velocidades de médias a altas para não se correr o risco de ter perdas amostrais por possíveis intercorrências, como dor forte, grande fraqueza muscular ou limitações de amplitude de movimento, o que poderia impedir que os indivíduos com alguma limitação articular não conseguissem participar do estudo. (PREIS et al., 2006).

A avaliação isocinética foi realizada em ambos os membros inferiores e, quando se comparou o pico de torque entre os membros inferiores direito e esquerdo, verificou-se que os valores eram muito próximos. O presente estudo está em concordância com o estudo de Aquino, que avaliou isocineticamente o torque dos músculos flexores e extensores dos joelhos de 26 mulheres idosas sem afecções do sistema musculoesquelético em membros inferiores, numa velocidade angular de $60 \%$ s. Os resultados demonstraram não haver diferenças entre os valores do torque máximo do lado dominante (D) e do lado não dominante (ND). Esses dados foram demonstrados tanto para o movimento flexor quanto para o extensor. (AQUINO et al., 2002).

$\mathrm{Na}$ comparação do pico de torque dos músculos extensores de joelho, dos indivíduos nas diferentes faixas etárias, tanto na amostra masculina quanto na feminina (nas faixas etárias de 60-69 e 70-79 anos), é possível observar que, quanto maior a velocidade, menor o pico de torque. Já nas amostras dos indivíduos de ambos os sexos com mais de oitenta anos de idade, não é possível estabelecer uma relação nesses parâmetros, pois o número de sujeitos é muito pequeno (dois indivíduos do sexo masculino e dois do sexo feminino).

Tanto a comparação entre grupos flexores bilaterais quanto a comparação entre extensores bilaterais podem apresentar algum desequilíbrio, porém considerado normal, entre o membro dominante e o não dominante de até $10 \%$. (DVIR, 2002). No presente estudo foi possível observar que, na comparação entre os membros inferiores direito e esquerdo, os desequilíbrios somente ultrapassaram $10 \%$ na amostra com mais de oitenta anos, no sexo masculino, o que não é significativo estatisticamente por ser esta amostra composta somente por dois indivíduos. Na média do pico de torque dos indivíduos do sexo masculino é possível verificar a significância do movimento de flexão a $240^{\circ}$. Ao comparar a média entre os diferentes gêneros, verifica-se que nos indivíduos do sexo masculino os picos de torque estão mais elevados.

A literatura descreve que próximo aos oitenta anos de idade são perdidos cerca de 40 a $50 \%$ da força muscular. Como nossa amostra apresentou um número muito pequeno de indivíduos acima de oitenta anos, limitou a comparação sobre esta maior perda da força. Contudo, apesar do número de dois indivíduos do sexo masculino e dois do feminino, na amostra no sexo masculino nas diferentes faixas etárias é possível observar que existe uma perda de aproximadamente $50 \%$ da força entre a faixa etária de sessenta e a de oitenta anos No sexo feminino esta diferença não se apresenta. 
O desempenho da força apresenta o seu pico entre vinte e trinta anos; após esse período permanece relativamente estável ou diminui ligeiramente durante os vinte anos seguintes. (CALMELS; MINAIRE, 1995; GRIFFIN et al., 1993).

No presente estudo verificou-se que nos indivíduos com diagnóstico de doenças osteoarticulares existe uma diminuição do pico de torque em comparação com os que não possuem doença osteoarticular (nas velocidades de $120^{\circ}$ de flexão e extensão e $180^{\circ}$ de extensão na amostra masculina e $120^{\circ}$ e $180^{\circ}$ de flexão e extensão na amostra feminina). Nas demais velocidades essas diferenças não se manifestaram.

Houve limitações para comparar os valores do presente estudo em razão de que se apresentou, em alguns resultados, falta de relação entre os dados apresentados, sobretudo quando se compara $o$ pico de torque relacionando-se com a velocidade utilizada, pois, quanto maior a velocidade, menor deveria ser o pico de torque.

Além do reflexo doloroso inibitório, quando não são geradas curvas uniformes, a presença de alterações unilaterais da anatomia, doença em que a fadiga é precoce, e baixo limiar de excitabilidade de órgão tendinoso de Golgi (OTG) são fatores que, individualmente ou somados, podem ser independentes do empenho do sujeito. (PREIS et al., 2006).

Borges, em 1989, avaliou flexores e extensores de joelho de 280 sujeitos de ambos os sexos, comparando mulheres e homens em três velocidades. Houve um declínio entre as idades de vinte e trinta anos em homens e quarenta e cinquenta anos nas mulheres. Houve também um outro declínio no momento, para ambos os sexos, entre as idades de sessenta e setenta anos. Não houve diferenças estatisticamente significativas entre os membros direito e esquerdo. (DVIR, 2002).

O decréscimo da função dos músculos quadríceps (Q) e isquiotibiais (IT) apresenta-se potencializado na população idosa portadora de osteoartrose de joelhos. Davini e colaboradores observaram interações entre o sexo e a força muscular. De acordo com os autores, indivíduos idosos do sexo masculino apresentaram maior força muscular do que os do sexo feminino. Isso poderia ser explicado pelas diferenças entre a composição e o tamanho corporal existente entre indivíduos do sexo masculino e feminino, as quais se mantêm com o envelhecimento. (DAVINI; NUNES, 2003).

A força dos músculos extensores é maior que a força dos músculos flexores. Comparando-se o pico de toque em pacientes de diferentes idades, observou-se que os valores da força para os extensores do joelho eram, na média geral, a metade do valor encontrado para os extensores. (CAPODAGLIO; PAOLO et al., 2008).

$\mathrm{O}$ valor da força muscular verificado nesta pesquisa foi significativamente maior nos indivíduos do sexo masculino do que nos do sexo feminino, independentemente da velocidade angular envolvida. Avaliando-se o torque de joelho-extensor por meio do dinamômetro, observou-se que existe uma maior ativação voluntária e que a força muscular foi maior nos 
indivíduos do sexo masculino. (RUITER; CORNELIUS, 2007).

Dentro do contexto avaliado, é importante ressaltar as limitações do presente estudo, tanto no número amostral como pelo fato de que indivíduos do sexo masculino estavam representados por um número menor. Também é relevante a baixa representatividade de indivíduos com mais de oitenta anos de idade, o que acabou limitando a comparação com as outras faixas etárias estudadas.

\section{Conclusão}

Tendo como base a avaliação de músculos flexores e extensores de joelho, foi possível estabelecer as seguintes conclusões: a força muscular (torque muscular) é maior nos indivíduos do sexo masculino; os músculos extensores do joelho são mais fortes; os flexores não apresentaram relação entre velocidade e pico de torque. A presença de doença osteoarticular, sobretudo nas mulheres, pode ter influenciado no pico de torque dos indivíduos do sexo feminino.

\section{Evaluate the muscular force of flexors and extensors of knee in socially active aged individuals}

\begin{abstract}
The aging brings obtains many phisiological manifestations, amongst these, the loss of muscular force, as well as the ability of the muscle to exerting force quickly. From the 60 years old this loss becomes more severe and is responsible for limitations in the functionality, deficits of balance and risk of falls. This research had as objective to evaluate the muscular force (muscular torque) of flexors and extensors of knee in socially active aged individuals. Partici-
\end{abstract}

pants: 100 socialy active aged individuals with age between 60 and 87 years old had participated of the research, of both sorts, that frequented the regional Center of Applied Studies to third age of the University of Passo Fundo, in the city of Passo Fundo, in the period of March to June of 2007, being excluded of the study the individuals that presented cognitive defict and that did not obtain the flexion of knee. Methodology: For the accomplishment of this research the isocinethic dynamometer Biodex Multi Joint 3 was used, in the speeds of $120^{\circ}$, $180^{\circ}$ and $240^{\circ}$, in the movements of flexion and extension. Initially the participants had carried through a heating of 5 minutes in ergometric bicycle, and after they had been submitted to a series of three repetitions of movements, being considered the average of the three repetitions. The evaluations had happened at the Laboratory of Biomechanics in the College of Physical Education and Physioterapy at the University of Passo Fundo - UPF. In our study it was possible to observe that in the comparison between the lower limbs right and left, the muscular unbalance had only exceeded $10 \%$ in the sample with more than 80 years, in the masculine sex, what we cannot only consider significant due to this sample to be composed for two individuals in each sort. In the average of the peak of torque for the individuals of the masculine sex $240^{\circ}$ with $p<0,03$ is possible to verify the significance of the flexion movement, 0,03 when analyzed all the sample, and for the individuals of 60-69 years in the extension movement $120^{\circ}$ with $p<0,04$. One comparing the different sorts is verified that the individuals of the masculine sex, they are the raised peaks of torque more elevated. The muscular force (muscular torque) is bigger in the individuals of the masculine sex; the extensors muscles of the knee (quadriceps) are strongest. The flexors muscles not present relacion velocity peak of tork. The presence of osteoarticular illness, mainly in the woman, can be influenced in the lesser peak of torque of individuals.

Key words: Aging. Muscle Strength. Torque. Muscle Strength Dynamometer. 


\section{Referências}

AQUINO, M. A. et al. Isokinetic assessment of knee flexor/extensor muscular strenght in ederly women. Revista do Hospital das Clínicas, São Paulo, v. 57, n. 4, p. 131-134, 2002.

CALMELS, P.; MINAIRE, P. A. Review of the role of the agonist/antagonist muscle pairs ratio in rehabilitation. Disability and Rehabilitation, v. 17, n. 6, p. 265-276, 1995.

CAPODAGLIO, Paolo et al. Strength characterization of knee flexor and extensor muscles in prader-willi and obese patients. BMC Musculoskeletal Disorders, Deutschland, p. 10-47, 2008.

CAROMANO, F. A.; TSAI, C. Estudo comparativo do desempenho em testes de força muscular entre indivíduos jovens e idosos através da miometria. Revista Fisioterapia da Universidade de São Paulo, São Paulo, v. 6, n. 1 , p. 101-112, 1999.

DAVINI, R.; NUNES, C. V. Alterações no sistema neuromuscular decorrentes do envelhecimento e o papel do exercício físico na manutenção da força muscular em indivíduos idosos. Revista Brasileira de Fisioterapia, São Carlos - SP, v. 7, n. 3, p. 201-207, 2003.

DVIR, Z. Isocinética: avaliações musculares, interpretações e aplicações clínicas. Barueri: Manole, 2002.

GRIFFIN, J. W. et al. Eccentric muscle performance of elbow and knee muscle groups in untrained men and women. Med. Sci. Sports. Exerc., v. 25, n. 8, p. 936-944, 1993.

LYNCH, N. A. et al. Muscle quality 1. Ageassociated differences between arm and leg muscle groups. J. Appl. Physiol., v. 86, p. 188-194, 1999.

PREIS, C. et al. Utilização da dinamometria isocinética como recurso de avaliação no complexo joelho. Revista Fisioterapia Brasil, São Paulo, v. 80, n. 10, p. 6-10, 2006.
MORELLI,J. G. et al. Fisioterapia geriátrica: a prática da assistência ao idoso. São Paulo: Manole, 2004.

RUITER, C. J.; GOUDSMIT, J. F. A.; TRICHT, J. A. The isometric torque at which knee-extensor muscle reoxygenation stops. Official Journal of the American College of Spots Medicine, v. 39, n. 3, p. 443-452, 2007. 